\title{
Fundamental Research on Hydraulic Systems Driven by Alternating Flow
}

\author{
Ioan-Lucian Marcu ${ }^{1}$, Daniel-Vasile Banyai ${ }^{1}$ \\ ${ }^{1}$ Technical University of Cluj-Napoca, Department of Mechanical Engineering, B-dul Muncii Nr. 103-105 Cluj-Napoca \\ Romania
}

Correspondence to: Lucian.Marcu@termo.utcluj.ro

\begin{abstract}
This paper presents a new approach to rotary hydraulic systems, and the functional principles of rotary hydraulic systems that can work using alternating flows. Hydraulic transmissions using alternating flows are based on bidirectional displacement of a predefined volume of fluid through the connection pipes between the alternating flow, the pressure energy generator and the motor. The paper also presents some considerations regarding the basic calculation formulas, the design and testing principles for a hydraulic motor driven by alternating flow, and also a three-phase rotary hydraulic motor.
\end{abstract}

Keywords: hydraulic, alternating flow, star or delta connection.

\section{General considerations}

All known methods of power transmission by a fluid, and their applications, are based on continuous pressure and flow circulation, which are achieved by the pump and collected by final elements (actuators), and the fluid is considered incompressible.

In conventional hydraulic transmissions, the fluid performs a unidirectional motion between the energy converters in the power transmitting process. In hydraulic transmissions driven by alternating flow, the fluid executes an alternating periodical motion between the energy converters. In the case of a hydraulic system in which every working volume of an alternating motor is connected independently, by a phase pipe, with the corresponding working volume of a generator, any modification to the volume of the generator will produce an alternating flow and pressure transmitted along the phase line to the motor.

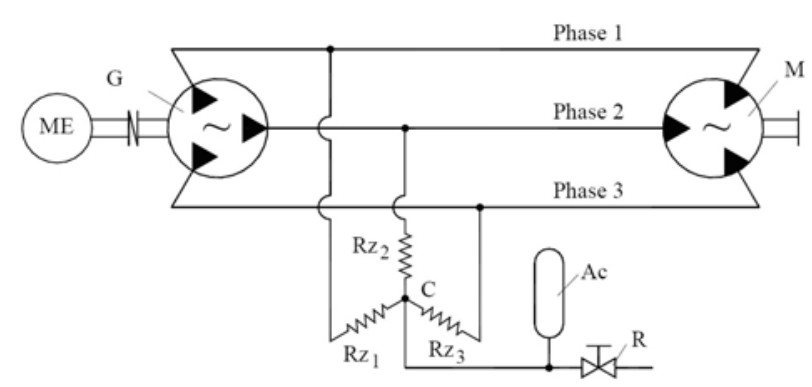

Figure 1: Principle schema of a rotary hydraulic system with alternating flows and pressure drive
Generally, a hydraulic transmission driven by a rotary alternating flow consists of a generator of alternating flows and pressures $(\mathrm{G})$ and a motor $(\mathrm{M})$. The connection between them is realized by a number of pipes equal to the number of phases (Phase 1, Phase 2 and Phase 3), the pipes being filled with fluid at a certain (pre-established) pressure, Figure 1. When the system is functioning, the pressure and the flow within each pipe varies in a sinusoidal way, around an average value [1].

In order to have proper functioning, the average pressure from each pipe must necessarily have the same value and have a constant value in time. To obtain the correct functionality, we therefore either create from the beginning a pressure in each phase that is higher than the maximum value of the amplitude, or we let the pressure modify itself while it is functioning.

This result is obtained by using a series of hydraulic resistances $\left(\mathrm{Rz}_{1}, \mathrm{Rz}_{2}, \mathrm{Rz}_{3}\right)$, rigorously calculated, which interconnect all the phases, and also a hydraulic accumulator (Ac) connected to them at point C, Figure 1. The resistances must eliminate the maximum average pressure rising value in one second, when the diminution of the flow amplitude from a phase does not exceed $1 \%$.

The presence of the accumulator maintains the pressure in a connection approximately constant at all time, being able to take over the oil surplus from the dilatations, and at the same time to supplement any oil loses. In the case of slow working cycles, as in this case, the correct transformation for the accumulator is the isotherm transformation, which leads us, 
knowing also the system pressure, to the necessary volume of the hydraulic accumulator.

This hypothesis was verified using an experimental stand consisting of a three-phase hydraulic transmission driven by an asynchronous alternating flow.

\section{Design principles of hydraulic energy converters driven by alternating flow}

As presented in Figure 1, a hydraulic transmission driven by alternating flow is a closed circuit that does not require a tank for the working fluid.

The generator was a modified axial piston pump (wobble-plate type) with three active pistons placed at angles of $120^{\circ}$, which allows variation of the plate angle, thus obtaining different strokes for the pistons.

A hydraulic rotary motor driven by alternating flow was realized using the principles presented in Figures 2a and 2b. [4]

The simplest solution for the construction of a rotary hydraulic motor working with alternating flow is to use in the same assembly three different oscillating motors with a gear rack (see elements 1 and 3 in Figure $2 \mathrm{a}$ and $2 \mathrm{~b}$ ). Because each piston moves independently, corresponding to its phase pipe, each gear wheel must have the possibility to act individually on the output shaft. The correct solution for this is to use an intermediary element, e.g. a drawn cup roller clutch, which provides a unidirectional rotation movement (see element 2 in Figures $2 \mathrm{a}$ and $2 \mathrm{~b}$ ).

In this construction, the three-phase generator provides an alternating flow only for the active stroke, and the retraction stroke of the motor pistons (the idle stroke) must be made also using an alternating flow provided by the generator, taking into account that each piston movement of the generator has a phase alteration with an angle of $120^{\circ}$. If this solution is taken into consideration, we can obtain two separate ways to interconnect for the working volumes of the star or delta motor.

Figures $2 \mathrm{a}$ and $2 \mathrm{~b}$ show that each phase pipe (line) is connected individually to a working volume of the motor cylinders, in this way providing the active stroke of the pistons. The working volumes (chambers) of the cylinders are interconnected.

In this way, if we consider that the movement of each piston has a phase difference of $120^{\circ}$, the advance of one motor piston will generate a similar flow in the star connection, which provides the retraction stroke (the idling stroke) of the next two pistons. A characteristic of the star connection is that the sum of the alternating flows in the connection point is theoretically zero, Figure 2a.

In the case of the delta connection, each first working volume of a cylinder, which provides the active stroke, is connected with the second working volume of the next phase cylinder, providing in this way its retraction stroke (the idling stroke); the pressures in these chambers are considered, of course theoretically, to have the same values, Figure $2 \mathrm{~b}$.

According to these functioning principles, a prototype of a rotary hydraulic three-phase motor was realized, working with alternating flows, Figures 3a and $3 \mathrm{~b} .[2]$

In principle, the designed rotary hydraulic motor consists of three associated hydraulic double end cylinders, with small dimensions, which act individually to an output shaft, thus obtaining a continuous rotational movement of the shaft.

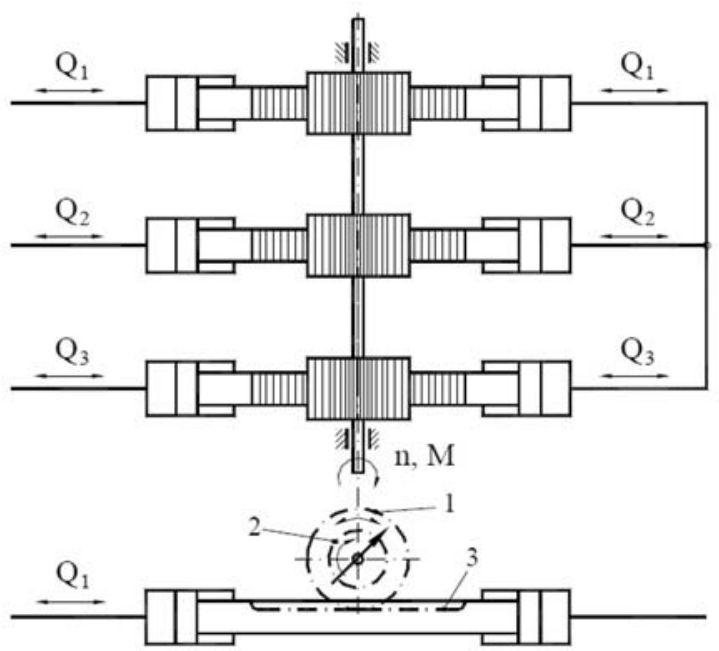

a) star interconnection

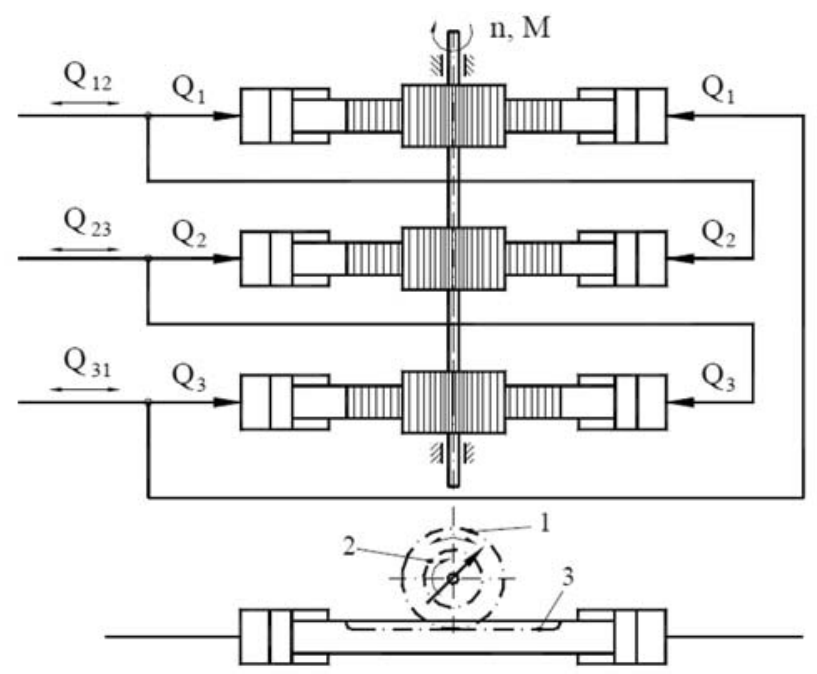

b) delta interconnection

Figure 2: Functioning principle of a rotary hydraulic motor driven by alternating flow 


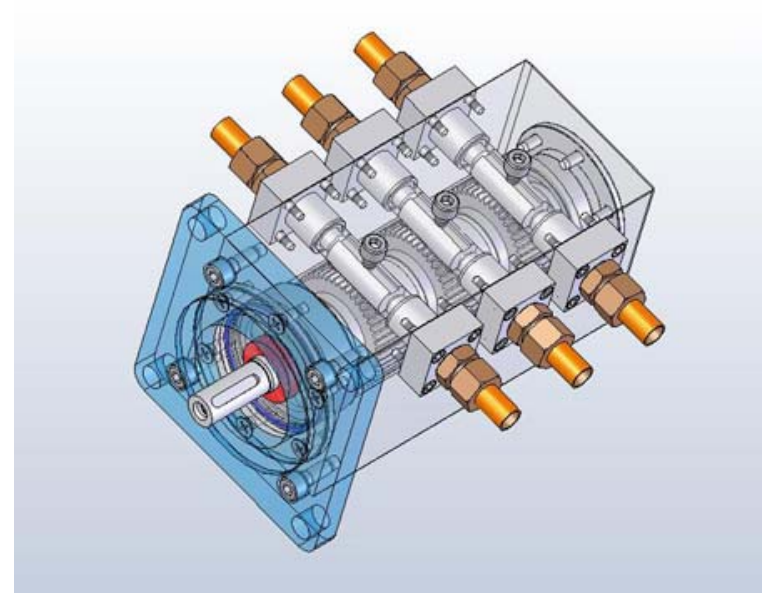

a)

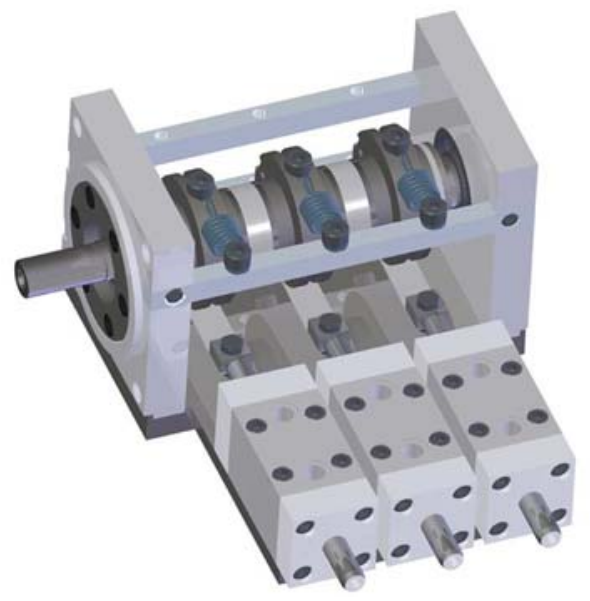

b)

Figure 3: The design of the three-phase rotary hydraulic motor driven by alternating flow

The hydraulic motor was designed in two versions. The compact model, Figure 3a, has gear rack conversion mechanisms associated to each small hydraulic cylinder group. Figure 3b presents an experimental model of the rotary hydraulic motor driven by alternating flow with a pulley and elastic steel sheet conversion mechanism. Each designed version contains three double end hydraulic cylinders which individually actuate some pulleys (or gear), having drawn cup roller clutches mounted inside, and in this way the rotational motion and also the torque are transmitted unidirectionally to the output shaft of the motor.

\section{Basic calculation}

We assume that the governing equations for the instantaneous flow and pressure are:

$$
Q_{i}=Q_{a \max } \cdot \sin \left(\omega t+\phi_{0}\right)
$$

And for the pressure:

$$
p_{i}=p_{s t}+p_{a \max } \cdot \sin \left(\omega t+\phi_{0}\right)
$$

in which: $\left(Q_{i}\right)$ is the instantaneous flow in $\left[\mathrm{m}^{3} / \mathrm{s}\right]$, $\left(Q_{a \max }\right)$ is the alternating flow amplitude in $\left[\mathrm{m}^{3} / \mathrm{s}\right]$, $\left(p_{i}\right)$ is the instantaneous pressure in $\left[\mathrm{N} / \mathrm{m}^{2}\right],\left(p_{s t}\right)$ is the initial static pressure of the system in $\left[\mathrm{N} / \mathrm{m}^{2}\right]$, $\left(p_{a \max }\right)$ is the alternating pressure amplitude in $\left[\mathrm{N} / \mathrm{m}^{2}\right],(\omega)$ is the angular frequency in $[\mathrm{rad} / \mathrm{s}],(t)$ is the time in $[\mathrm{s}]$, and $\left(\phi_{0}\right)$ is the initial phase angle in $[\mathrm{rad}]$.

Making a complete analysis of a hydraulic system working with alternating flow requires that we take into account some parameters that define the pressure drop along the line due to the friction, inertia and elasticity (compressibility) of the fluid, and also the fluid leaks.

The instantaneous pressure drop due to the friction along the line will be:

$$
\Delta p_{i R}=R_{f} \cdot Q_{a \max } \cdot \sin \left(\omega t+\phi_{0}\right)
$$

where $\left(R_{f}\right)$ is the flow resistance, in $\left[\mathrm{N} \cdot \mathrm{s} / \mathrm{m}^{5}\right]$.

Eq. 3 demonstrates the fact that the instantaneous flow is in phase with the pressure drop through the resistance.

The pressure drop due to fluid inertia is:

$$
\Delta p_{i L}=\omega \cdot L \cdot Q_{a \max } \cos \left(\omega t+\phi_{0}\right)
$$

where $(L)$ is the fluid inertia, in $\left[\mathrm{N} \cdot \mathrm{s}^{2} / \mathrm{m}^{5}\right]$.

The phase vector of the inductive pressure drop is advanced by $90^{\circ}$ in comparison to the instantaneous flow.

If we consider the elasticity of the transmitting medium, the capacitive pressure drop is:

$$
\Delta p_{i C}=-\frac{Q_{a \max }}{C_{h} \cdot \omega} \cdot \cos \left(\omega t+\phi_{0}\right)
$$

where $\left(C_{h}\right)$ is the hydraulic capacity $\left[\mathrm{m}^{5} / \mathrm{N}\right]$.

It can be noted that the vector of the capacitive pressure drop is $90^{\circ}$ behind the instantaneous flow.

\section{Testing principles and experimental results}

The functioning prototype was tested in the idling state and in the loaded state. The load was obtained by using a braking device connected to the output shaft and actuated by a small hydraulic cylinder. This way, we can obtain different values for the loading torque. The preliminary experimental data, representing the functional parameters, was obtained taking into account the monitoring protocol, the disposition of the sensors, and using a data acquisition board, Figure 4. 


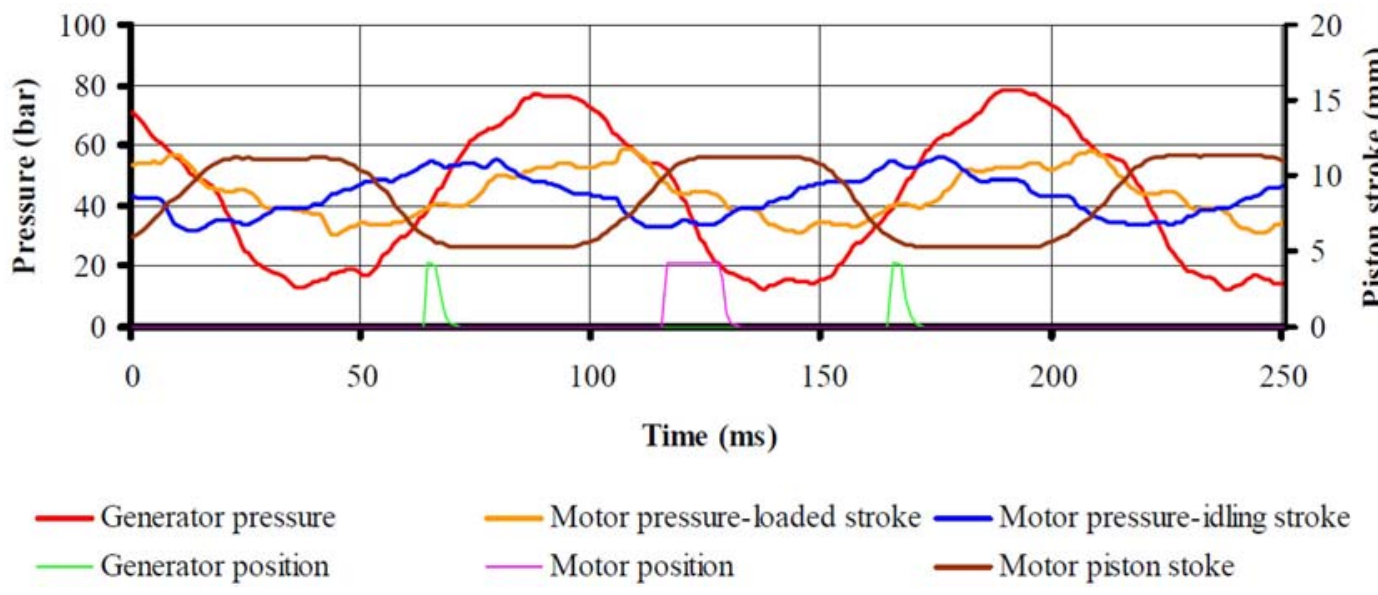

Figure 4: Pressures, motor piston stroke and angular speed evolution

In order to acquire the experimental data during the testing process we had to control the mechanical and hydraulic parameters precisely, using proximity sensors mounted on the rotation shafts, pressure sensors mounted on representative points of the hydraulic pipes, and displacement sensors mounted on the motor cylinder pistons. [3]

A series of functional parameters of the hydraulic system driven by alternating flow was obtained directly, as: angular speed of the shafts, generator and motor; pressures (on the generator, on the motor loaded, on the motor - idle, in the phase line pipes or in the interconnection pipes); and the piston strokes of the motor.

Some of the diagrams were obtained indirectly, using recorded data referring to: transmitted torques and phase alteration between alternating flow and harmonic pressure or the rotational position of the generator and motor shafts.

\section{Conclusion}

The objective of this research was to study a new approach to hydraulic drives, in which the pressure and flow is not continuously transmitted between the energy converters (pumps and motors). The paper describes the construction principles of hydraulic systems driven by alternating flow.

Within these systems, the active stroke of the pistons is produced by the flow of pressurized fluid from the generator, while, for the retraction stroke, a supplementary connection to a pressure generator is necessary, working in the opposite phase to the first. This means that the pistons can be interconnected in star or delta configuration.

The experimental results, combined with the mathematical model of this system, demonstrate a way to adjust, during the functioning, several input parameters (e.g. the initial static pressure and the angular speed of the generator), in order to obtain the anticipated output values of some parameters, or whether the system load is modifying.

Knowing the limits of this system, the next development step is automatic control of the main mechanical and hydraulic output parameters.

\section{References}

[1] Constantinescu, G.: Theory of sonics. Bucureşti : Published by Romanian Academy, 1985 (in Romanian).

[2] Marcu, I.-L.: Researches and contributions regarding the improving of the acting systems functioning with pressure waves. $\mathrm{PhD}$. Thesis, ClujNapoca, 2004.

[3] Marecu I.-L.: Functional parameters monitoring for an alternating flow driven hydraulic system. In IEEE Proceedings of International Conference AQTR, Cluj-Napoca, 2008, Tome III, p. 239. ISBN 978-1-4244-2576-1.

[4] Pop, I., a.o.: Sonics Applications. Experimental Results. Ed. Performantica, Iaşi, 2007 (in Romanian). ISBN 978-973-730-391-2. 